\title{
The effect of sex and maturation on liver docosahexaenoic acid status in rats
}

\author{
S. P. Hoile ${ }^{1}$, E. Penketh ${ }^{2}$, K. A. Lillycrop ${ }^{2}$ and G. C. Burdge ${ }^{1}$ \\ ${ }^{1}$ Faculty of Medicine and ${ }^{2}$ Faculty of Natural and Environmental Sciences, University of Southampton, Southampton, \\ SO16 6YD
}

Women aged less than 40 have higher docosahexaenoic acid $(22: 6 n-3)$ status ${ }^{(1)}$ and convert more $18: 3 n-3$ to longer chain $n-3$ polyunsaturated fatty acids (PUFA) than men ${ }^{(2)}$. Female rats also have higher liver and plasma phospholipid 22:6n-3 status than males ${ }^{(3)}$. Greater PUFA biosynthesis in women involves increased conversion of $22: 5 n-3$ to $22: 6 n-3^{(4)}$, and female sex hormones have been shown to increase plasma $22: 6 n-3$ concentration and to up-regulate $22: 6 n-3$, although the precise mechanism remains unclear ${ }^{(5)}$. It is not known when in the life course the sexual divergence in 22:6n-3 status and PUFA biosynthesis is induced. We hypothesised that puberty is the key period in which capacity for 22:6n-3 synthesis and status increases in females. To test this, we compared the liver fatty acid composition of juvenile and adult male and female rats.

Pregnant and lactating Wistar rats were fed a semi-purified diet (AIN93G) throughout gestation. Offspring were either weaned on postnatal d28 onto AIN93M or killed on postnatal d70 ( $n 7$ males or females per age group, one male or female per litter). Livers were collected, snap frozen and the fatty acid composition of liver phosphatidylcholine (PC) was measured by gas chromatography ${ }^{(6)}$.

There was a significant interaction effect of age*sex on the proportion of $18: 3 n-3(P=0.027)$ and 20:5n-3 $(P=0.009)$ in liver PC such that the proportions of these fatty acids were higher in $\mathrm{d} 28$ females and in d70 males and females compared to d28 males (Figs. 1 and 2 ). There was a significant effect of age $(P<0.001)$, but not sex, on proportion of $22: 5 n-3$ in liver PC such that it was lower d70 liver compared to d28 (Fig. 3). There was a significant age*sex interaction $(P=0.001)$ on proportion of 22:6n-3 in liver PC. The proportion of 22:6n-3 was significantly greater at d28 compared to d70 irrespective of sex. The proportion of 22:6n-3 in liver PC was higher in adult females than males, but did not differ between sexes at d28 (Fig. 4).

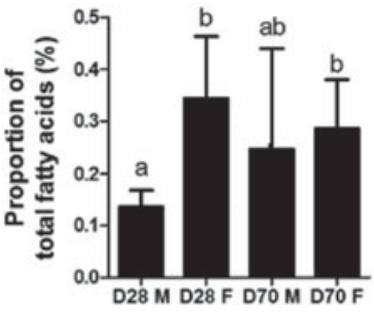

Fig. 1. Liver PC $18: 3 n-3$.

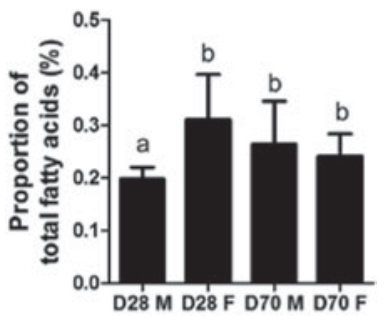

Fig. 2. Liver PC 20:5n-3.

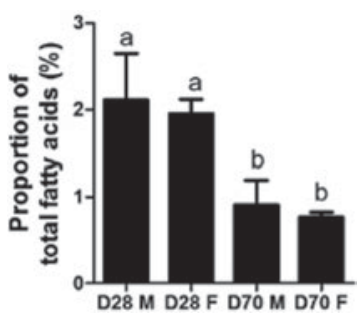

Fig. 3. Liver PC 22:5n-3.

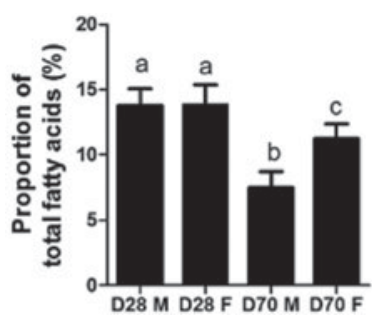

Fig. 4. Liver PC 22:6n-3.

Values are mean (SD). Data were analysed by two-way ANOVA with sex and age as fixed factors, with Bonferroni's post hoc test. Means with different letters differed significantly $(P<0.05)$.

These findings show that both age and sex affect the proportions of $n-3$ PUFA in liver in a manner contingent on the fatty acid species. As in human plasma $\mathrm{PC}^{(7)}$, the proportion of $20: 5 n-3$ in rat liver PC appears to be related to $18: 3 n-3$ status. Synthesis of $22: 5 n-3$ and $22: 6 n-3$ appeared to be independent of $20: 5 n-3$ status and to be down-regulated in adults compared to juveniles. The sex difference in the proportion of $22: 6 n-3$, but not $22: 5 n-3$, in adults is consistent with greater conversion of $22: 5 n-6$ to $22: 6 n-3$ in women than men ${ }^{(1-4)}$ and with the suggestion that these metabolic reactions are regulated independently of the conversion of $18: 3 n-3$ to $20: 5 n-3{ }^{(8)}$. Our findings support the suggestion that puberty represents an important influence on PUFA metabolism which acts through several regulatory loci.

1. Burdge GC \& Wottoon SA (2002) Br J Nutr 88, 411-20.

2. Burdge GC, Jones AE \& Wottoon SA (2002) Br J Nutr 88, 355-363.

3. Childs CE, Romeu-Nadal M, Burdge GC et al. (2008) Proc Nutr Soc 67, 19-27.

4. Pawlosky R, Hibbeln J, Lin Y et al. (2003) Br J Nutr 90, 993-995.

5. Giltay EJ, Gooren LJ, Toorians AW et al. (2004) Am J Clin Nutr 80, 1167-74.

6. Burdge, GC et al. (2000) Br J Nutr 84, 781-787.

7. Burdge \& Calder (2006) Nutr Res Rev 19, 26-52.

8. Sprecher H, Baykousheva S (1994) World Rev Nutr Diet 75, 26-9. 